\title{
Branching of the $p$-nitrophenol (PNP) degradation pathway in burkholderia sp. Strain SJ98: Evidences from genetic characterization of PNP gene cluster
}

Surendra Vikram¹, Janmejay Pandey ${ }^{1,2}$, Nidhi Bhalla', Gunjan Pandey ${ }^{1,3}$, Anuradha Ghosh ${ }^{1,4}$, Fazlurrahman Khan ${ }^{1}$, Rakesh K Jain ${ }^{1 \wedge}$ and Gajendra PS Raghava ${ }^{1 *}$

\begin{abstract}
Aerobic microbial degradation of $p$-nitrophenol (PNP) has been classically shown to proceed via 'Hydroquinone (HQ) pathway' in Gram-negative bacteria, whereas in Gram-positive PNP degraders it proceed via 'Benzenetriol (BT) pathway'. These pathways are characterized by the ring cleavage of HQ and BT as terminal aromatic intermediates respectively. Earlier reports on PNP degradation have indicated these pathways to be mutually exclusive. We report involvement of both ' $\mathrm{HQ}$ ' and 'BT' ring cleavage pathways in PNP degradation by Burkholderia sp. strain SJ98. Genetic characterization of an $\sim 41 \mathrm{~Kb}$ DNA fragment harboring PNP degradation gene cluster cloned and sequenced from strain SJ98 showed presence of multiple orfs including $p n p C$ and $p n p D$ which corresponded to previously characterized 'benzenetriol-dioxygenase (BtD)' and 'maleylacetate reductase (MaR)' respectively. This gene cluster also showed presence of $P n p E 1$ and $P n p E 2$, which shared strong sequence identity to cognate sub-units of 'hydroquinone dioxygenase' (HqD). Heterologous expression and biochemical characterization ascertained the identity of PnpE1 and PnpE2. In in vitro assay reconstituted heterotetrameric complex of PnpE1 and PnpE2 catalyzed transformation of hydroquinone (HQ) into corresponding hydroxymuconic semialdehyde (HMS) in a substrate specific manner. Together, these results clearly establish branching of PNP degradation in strain SJ98. We propose that strain SJ98 presents a useful model system for future studies on evolution of microbial degradation of PNP.
\end{abstract}

Keywords: P-nitrophenol, Hydroquinone dioxygenase, PNP pathway, Burkholderia sp SJ98

\section{Introduction}

P-Nitrophenol (PNP) is one of the most thoroughly studied toxic environmental pollutants; it has been widely used in industries for chemical synthesis of dyes and plastics, resulting in high levels of PNP contaminations (Bhushan et al. 2000; Spain 1995). A number of Gram-positive and Gram-negative bacterial strains have been isolated and characterized for PNP degradation. Furthermore, a number of studies have shown biochemical characterization and elucidation of the catabolic pathway for PNP degradation. Results presented in these studies indicated that aerobic PNP degradation could

\footnotetext{
*Correspondence: raghava@imtech.res.in

Deceased

'Institute of Microbial Technology (CSIR), Sector 39 A, Chandigarh 160036 India

Full list of author information is available at the end of the article
}

proceed through one of the two exclusive and independent pathways (Shen et al. 2010; Chauhan et al. 2010; Kitagawa et al. 2004). PNP degradation pathway observed with Gram-positive bacteria e.g. Arthrobacter sp. strain JS443 (Jain et al. 1994; Perry and Zylstra 2007), Bacillus sphaericus strain JS905 (Kadiyala and Spain 1998), Rhodococcus opacus strain SAO101 (Kitagawa et al. 2004) and Rhodococcus sp. strain PN1 (Takeo et al. 2008) preferentially proceeds via formation of benzentriol (BT) as the terminal aromatic intermediate which undergoes ring cleavage for fission of the aromatic ring. On the other hand, Gram-negative bacteria e.g. Moraxella sp. strain A1 (Spain and Gibson 1991), Pseudomonas spp. (Liu et al. 2005; Wei et al. 2010) usually degrade PNP via formation of 'hydroquinone' (HQ) as the ring cleavage substrate. A few studies have indicated conversion of $\mathrm{HQ}$ into $\mathrm{BT}$, which is then used as the terminal ring cleavage 
substrate in the degradation of 4-hydroxybenzoate and $p$ nitrophenol by Candida parapsilosis CBS604 and Pseudomonas aeruginosa HS-D38 respectively (Figure 1A) (Eppink et al. 2000; Zheng et al. 2009).

As variations to the above generalization, a few studies have reported alternative PNP degradation pathways. Chauhan and co-workers reported PNP degradation in Arthrobacter protophormiae strain RKJ100 to proceed via conversion of BT into hydroxy-benzoquinone (HBQ), which is subsequently converted into benzoquinone (BQ) and HQ (Chauhan et al., 2000). In another study, Jain et al. (1994) reported accumulation of both $\mathrm{BT}$ and HQ with ${ }^{14} \mathrm{C}$ trapping studies (at $~ 88 \%$ and $8 \%$ respectively) during PNP degradation by Arthrobacter sp. strain JS443. However, HQ could not be degraded further by strain JS443 and therefore it was suggested to be a degradation byproduct and/or dead-end degradation product (Jain et al. 1994). Genetic characterization of PNP degradation gene cluster of strain JS443 ascertained the above observation (Perry and Zylstra 2007). Another study reported PNP degradation by Pseudomonas sp. strain WBC-3 to selectively proceed via $\mathrm{HQ}$. Noticeably, strain WBC-3 could also degraded 4-NC, wherein degradation selectively proceeds via formation of BT (Wei et al. 2010a). Based on these reports, it could be argued that microbial degradation of PNP proceeds via either BT or HQ branch of the pathway. Previously, we reported that Burkholderia sp. strain SJ98 (previously identified as Ralstonia sp. SJ98 (Samanta et al; 2000; Paul et al. 2008)); a Gram-negative bacterium, metabolizes PNP as sole source of carbon and energy. PNP induced culture of strain SJ98 degrade PNP with formation of BT and maleylacetate (MA) as major transformation intermediate, whereas 4-nitrocatechol (4-NC) and HQ are detected as transient intermediates (Chauhan et al. 2010). A $6.8 \mathrm{~Kb}$ DNA fragment cloned from genome of strain SJ98 showed presence of some of the genes involved in lower part of the PNP degradation pathway. Two genes viz., $p n p C$ and $p n p D$ showed highest sequence similarity with genes encoding for 1,2,3-benzenetriol dioxygenase (BtD) and maleylacetate reductase (MaR) respectively (Chauhan et al. 2010). Heterologous expression and biochemical characterization of these genes ascertained their identity.

In this study we have carried out further genetic characterization of PNP catabolic cluster/genes in strain

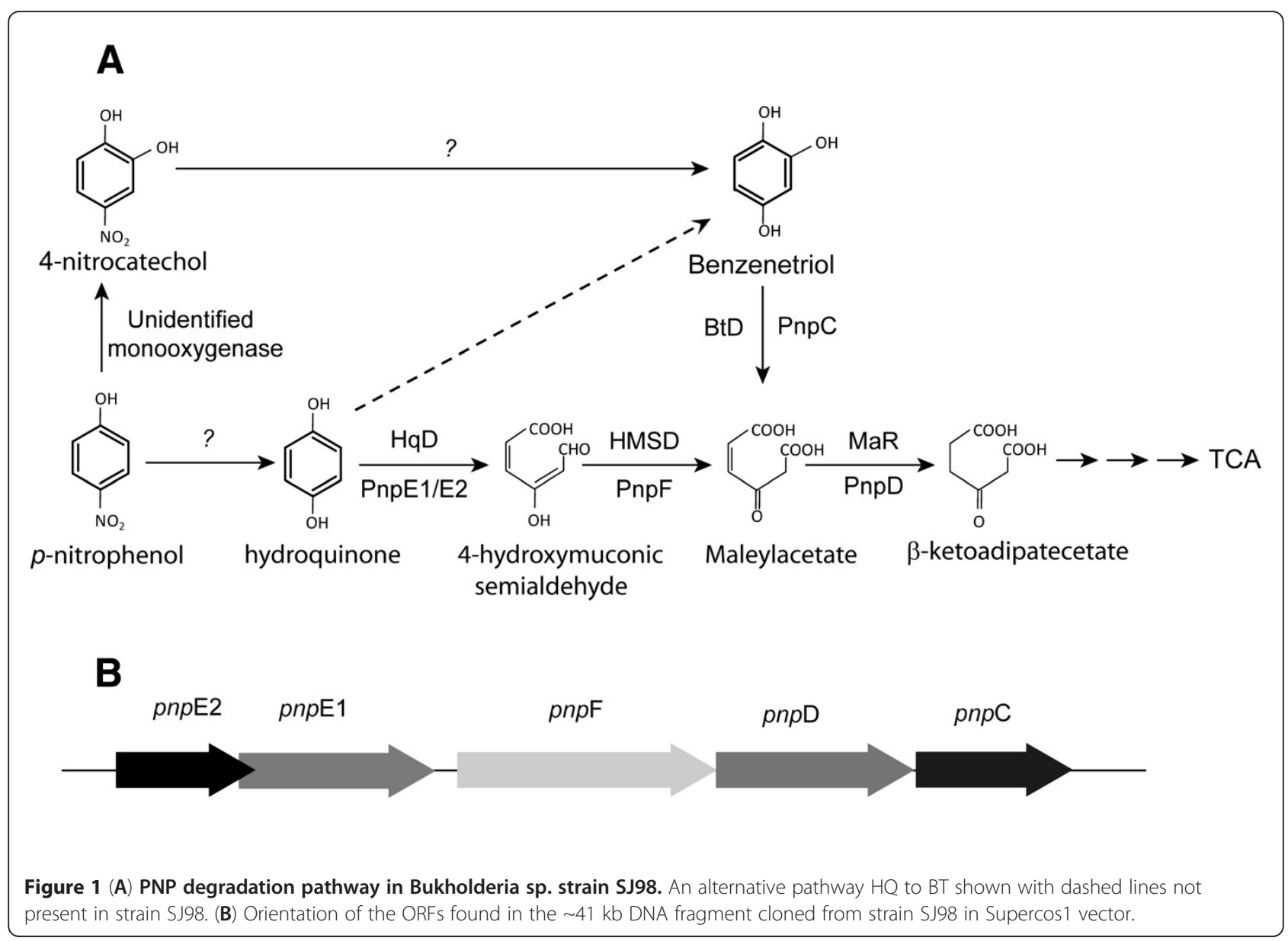


SJ98. Our observations indicate co-occurrence of functional genes, encoding for enzymes involved in both 'HQ and BT' ring cleavage pathways of PNP degradation, within the PNP degradation gene cluster of strain SJ98. Based on these genetic evidences together with earlier report by Chauhan et al. (2010) we propose that PNP degradation in strain SJ98 shows branching and proceed via both BT and HQ branches of catabolic pathway. Similar observation for involvement of both the branches of PNP degradation pathway has been recently reported in Pseudomonas sp. 1-7 (Zhang et al. 2012). To the best of our knowledge, Burkholedria sp. strain SJ98 along with Pseudomonas sp.1-7 is one of the first strains showing involvement of both the branches of PNP degradation pathway.

\section{Materials and methods}

Bacterial strains, plasmids, chemicals, media and culture conditions

Bacterial strains, plasmids and cosmid used in this study are listed in Table 1. Burkholderia sp. SJ98 was grown aerobically in Luria-Bertani (LB) medium or minimal medium (MM) prepared (Samanta el al. 2000) at $30^{\circ} \mathrm{C}$. Escherichia coli strains and recombinant clones were grown in LB medium at $37^{\circ} \mathrm{C}$. Filter sterilized antibiotics were added at a final concentration of $100 \mu \mathrm{g} \mathrm{ml}^{-1}$ (ampicillin) or $50 \mu \mathrm{g} \mathrm{ml} \mathrm{m}^{-1}$ (kanamycin) wherever required. Reagents and aromatic compounds used in this study were procured from Sigma Aldrich Chemical Co. (St. Louis, MO, USA). Media components were purchased from HiMedia Laboratories Pvt. Ltd. (Mumbai, India).
Nucleic acid isolation and genomic library construction Total genomic DNA of strain SJ98 was isolated and purified using Qiagen DNA Isolation Kit (Qiagen, Germany). Plasmid DNA was isolated with Qiagen Miniprep plasmid DNA Purification Kit (Qiagen, Germany) according to manufacturer's instructions. Total cellular RNA was isolated using Promega RNeasy Miniprep Kit (Promega, USA) following manufacturers protocol. For genomic library construction, restriction digestion fragments of Sau3AI-digested genomic DNA of strain SJ98 were ligated to $\mathrm{Bam} \mathrm{HI}$-digested linearized cosmid vector SuperCos-1 (Stratagene, USA). Subsequent packaging was carried out using Gigapack III gold packaging extract (Stratagene, USA). The packaged DNA was amplified up to 10 folds and transformed into electrocompetent E. coli DH5 $\alpha$ cells, using GenePulser Xcell ${ }^{\mathrm{TM}}$ Microbial Electroporation System (Bio-Rad Laboratories, USA). Transformed cells were spread plated onto LB- agar with ampicillin $(100 \mu \mathrm{g} / \mathrm{ml})$ and incubated at $37^{\circ} \mathrm{C}$ overnight.

\section{Genomic library screening and DNA sequence analyses} In order to screen the genomic library for PNP degrading gene cluster, a PCR amplicon ( $540 \mathrm{bp})$ corresponding to partial BtD gene amplified from genomic DNA of strain SJ98 (Chauhan et al., 2010), was used as radiolabeled DNA probe in colony hybridization analysis. Radio labeling of the amplicon was carried out using Megaprime DNA Labeling System (GE- Healthcare, USA). Positive clones obtained in colony hybridization assay were further confirmed with colony PCR and subsequently subjected to restriction digestion analysis for determining the size of the insert in each of these. One

Table 1 Bacterial strains and plasmids used in this study

\begin{tabular}{|c|c|c|}
\hline $\begin{array}{l}\text { Bacterial } \\
\text { strain/plasmid/cosmid }\end{array}$ & Characteristic & Source or reference \\
\hline \multicolumn{3}{|l|}{ Bacterial strains } \\
\hline $\begin{array}{l}\text { Burkholderia sp. } \\
\text { strain SJ98 }\end{array}$ & Wild type PNP degrading isolate & Lab stock and DSM $=23195$ \\
\hline E. coli $\mathrm{DH} 5 \mathrm{a}$ & $\begin{array}{l}\text { Host strain for cosmid cloning vector } \\
\text { Host strain for GateWay entry clone }\end{array}$ & Lab stock \\
\hline E. coli BL-21 Al & $\begin{array}{l}\text { Host strain for expression vector (LacY1DE3, } \\
\text { F_ompT hsdS, gal, dcm, ara-l) }\end{array}$ & Invitrogen Inc. CA- USA \\
\hline \multicolumn{3}{|l|}{ Plasmid/cosmid } \\
\hline SuperCos-1 & Cosmid cloning vector, Dual cos sites & $\begin{array}{l}\text { Agilent Technologies- } \\
\text { Genomics. CA, USA. }\end{array}$ \\
\hline pSJC88 & $\begin{array}{l}\text { Cosmid clone with } \sim 41 \mathrm{~Kb} \text { insert harboring PNP } \\
\text { degrading gene cluster of strain SJ98 }\end{array}$ & This study \\
\hline pDONR-201 & Gateway entry cloning vector & Invitrogen Inc. CA, USA \\
\hline pDEST-17 & Gateway expression vector & Invitrogen Inc. CA, USA \\
\hline pDest-pnpE1 & Expression clone with orf pnpE1 of strain SJ98 & This study \\
\hline pDest-pnpE2 & Expression clone with orf pnpE2 of strain SJ98 & This study \\
\hline
\end{tabular}


of these clones, designated as pSJC88, with $\sim 41 \mathrm{~Kb}$ insert was selected for further studies. Nucleotide sequence of the insert was determined using an $\mathrm{ABI}$ Model 377 DNA Sequencer and the Auto-Assembler program (Applied Biosystems, USA). Annotation of the above DNA sequence for ORF search and homology analyses of the translated nucleotide sequence was carried out using ORF finder and BLAST programs (http:// www.ncbi.nlm.nih.gov/). Pair-wise alignments and identity calculations were performed using NeedlmanWunsch global alignment algorithm (http://www.ebi.ac. uk/emboss/align). Vector NTI 10.0 (Invitrogen Technologies, USA) was used to generate the graphical output report of the annotated DNA fragment.

\section{Cloning and expression of pnpE1 and pnpE2}

ORFs $p n p E 1$ and $p n p E 2$ were individually amplified with high fidelity Pfu DNA polymerase (Fermentas, USA) using primer pair listed as pnpE1F, pnpE1R and pnpE2F, pnpE2R respectively (Table 2). Cosmid DNA isolated from pSJC88 was used as the reaction template. The standard PCR reaction mix $(25 \mu \mathrm{l})$ consisted of $100 \mathrm{ng}$ cosmid DNA, $0.2 \mathrm{mM}$ of each primer, $2.5 \mu \mathrm{l}$ of 10X PCR buffer, $1 \mu \mathrm{l}$ of $10 \mathrm{mM}$ dNTPs mix, and $1.25 \mathrm{U}$ of Pfu DNA polymerase (Fermentas, USA). The thermocycler program used for amplification of pnpE1 and pnpE2 was the following: (i) initial denaturation at $95^{\circ} \mathrm{C}$ for $5 \mathrm{~min}$; (ii) 10 cycles of denaturation at $95^{\circ} \mathrm{C}$ for $1 \mathrm{~min}$, primer annealing at $48^{\circ} \mathrm{C}$ for $30 \mathrm{~s}$ and fragment amplification $72^{\circ} \mathrm{C}$ for $1 \mathrm{~min}$; (iii) followed by 25 cycles of denaturation at $95^{\circ} \mathrm{C}$ for $1 \mathrm{~min}$, primer annealing at $63^{\circ} \mathrm{C}$ for $15 \mathrm{~s}$ and fragment amplification $72^{\circ} \mathrm{C}$ for $1.5 \mathrm{~min}$. A final extension was performed for $10 \mathrm{~min}$ at $72^{\circ} \mathrm{C}$. The resultant amplicons were analyzed on $1 \%$ agarose gel and later recombined with 'Gateway Cloning Technology' entry vector pDONR201 and destination expression vector pDEST17 (Invitrogen Technologies, USA) using BP and LR reactions respectively. The recombination cloning reactions were carried out according to the manufacturer's recommendation. The final expression plasmids pDEST-pnpE1 and pDEST-pnpE2 were confirmed by nucleotide sequencing and subsequently transformed into arabinose inducible strain of $E$. coli BL-21 (Invitrogen Technologies, USA). Expression clones harboring pDEST-pnpE1 and pDEST-pnpE2 were grown in LB medium containing ampicillin $\left(100 \mu \mathrm{g} \mathrm{ml}^{-1}\right)$ at $30^{\circ} \mathrm{C}$ to an $\mathrm{OD}_{600}$ of $\sim 0.4$ and then induced for $8-10 \mathrm{hrs}$ by adding $0.2 \%$ of Arabinose at $18^{\circ} \mathrm{C}$.

\section{Preparation of cell extracts and purification of recombinant PnpE1 and PnpE2}

$500 \mathrm{ml}$ culture of induced recombinant clones of pDESTpnpE1 and pDEST-pnpE2 were harvested by centrifugation, washed twice with ice-cold $1 \mathrm{X}$ Phosphate buffered saline (PBS) and then re-suspended in $25 \mathrm{ml}$ of cell lysis buffer $(50 \mathrm{mM} \mathrm{NaHPO}, 300 \mathrm{mM} \mathrm{NaCl}, 10 \%$ v/v glycerol and $10 \mathrm{mM}$ Imidazole; $\mathrm{pH} 8.0$ ) and lysed by ultrasonication. Soluble and insoluble fractions were separated through centrifugation at 20,000 g for $15 \mathrm{~min}$. The separated fractions were tested for presence of over-expressed recombinant PnpE1 and PnpE2. Supernatant obtained from lysate of pDEST-pnpE2 showed presence of overexpressed protein, therefore it was purified with affinity chromatography using Ni-NTA Agarose-superflow columns (Qiagen, Germany), pre-equilibrated with lysis buffer. His-tagged PnpE2 was eluted with a linear gradient of imidazole ranging from $10 \mathrm{mM}-200 \mathrm{mM}$ in the buffer above. Thereafter, the eluted fractions were dialyzed two times with $1 \mathrm{l}$ of buffer containing $50 \mathrm{mM} \mathrm{NaHPO}$, $300 \mathrm{mM} \mathrm{NaCl}, 10 \%$ glycerol ( $\mathrm{pH}$ 8.0). Purified protein fractions were stored with $20 \%$ glycerol at $-20^{\circ} \mathrm{C}$ for further use. Purity of the eluted protein samples were tested by SDS-PAGE analysis. Since, majority of the overexpressed PnpE1 was found to be present in inclusion bodies, a denaturation, dialysis, and refolding procedure was followed in order to purify PnpE1.

Table 2 Oligonucleotide primers designed and used in this study

\begin{tabular}{llll}
\hline Target ORF & Primer name & Primer sequence $\left(\mathbf{5}^{\prime} \rightarrow \mathbf{3}^{\prime}\right)$ & Application/purpose \\
\hline PnpE1 & pnpE1_F & GGGGACAAGTTGTACAAAAAGCAGGCTTAATGGGCCGACATCTGCAT & HqD Large subunit amplification \\
\hline & pnpE1_R & GGGGACCACTTTGACAAGAAAGCTGGGTATTAGAACGCGACCGGATA & \\
\hline PnpE2 & pnpE2-F & GGGGACAAGTTTGTACAAAAAAGCAGGCTTATGGAGACAGACATGCAA & HqD Small subunit amplification \\
\hline PnpE1 & pnpE2-R & GGGGACCACTTTGTACAAGAAAGCTGGGTATTACTGGATGCAGATGTC & \\
\hline & pnpE1-RT_F & TCTACGGCTGGGTCAATTC & HqD large subunit RT-PCR primer \\
\hline PnpE2 & pnpE1-RT_R & CTTCGTCACCCAGTCCTTC & HqD Small subunit RT-PCR primer \\
\hline pnpE2-RT_F & CGCATTACGTGATGTCCAAC & Partial BtD gene amplification \\
\hline PnpD & pnpE2-RT_R & GTTCACCGAGCCTTCGATA & \\
\hline
\end{tabular}




\section{Refolding and purification of PnpE1}

Insoluble fractions collected from the cell lysate of $E$. coli recombinant with pDEST-pnpE1 was resuspended in denaturing buffer $(50 \mathrm{mM} \mathrm{NaHPO}, 6 \mathrm{M}$ urea, $10 \mathrm{mM}$ imidazole, $300 \mathrm{mM} \mathrm{NaCl}, 5 \mathrm{mM} \beta$-mercaptoethanol (pH 8.0)) and incubated overnight at room temperature. Afterwards, the resuspended sample was centrifuged at $14,000 \mathrm{~g}$ for $30 \mathrm{~min}$ to separate the soluble and insoluble fractions. The soluble fraction was separated and then subjected to affinity chromatography based purification using Ni-NTA Superflow column (Qiagen, Germany) under denaturing condition according to the manufacturer's instruction. After elution with linear gradient of imidazole in denaturing buffer, PnpE1 was diluted 100 fold in the refolding buffer $(50 \mathrm{mM} \mathrm{NaHPO} 4$ 7.0, $10 \mathrm{mM}$ imidazole, $300 \mathrm{mM} \mathrm{NaCl}, 5 \mathrm{mM}$ BME and $10 \%$ glycerol ( $\mathrm{pH} 8.0)$ ) and allowed to refold at $4^{\circ} \mathrm{C}$ for $12 \mathrm{~h}$. Refolded PnpE1 was again eluted through Ni-NTA Superflow beads (Qiagen, Germany) in the native purification buffer following the manufacturer's instructions. The refolded PnpE1 was centrifuged at $14,000 \times \mathrm{g}$ for 30 minute to remove residual aggregate. PnpE1 was dialyzed twice with $1150 \mathrm{mM} \mathrm{NaHPO}_{4}$ buffer, $\mathrm{NaCl}$ $300 \mathrm{mM}, 10 \%$ Glycerol and stored at $-20^{\circ} \mathrm{C}$ in $20 \%$ glycerol.

\section{Reconstitution of hydroquinone dioxygenase (HqD) with PnpE1 and PnpE2}

In order to reconstitute the $\mathrm{HqD}$ activity, the purified recombinant proteins PnpE1 and PnpE2 were mixed in different molar ratios ranging from 3:1 to $1: 3$ in $1 \mathrm{ml}$ of $50 \mathrm{mM}$ phosphate buffer @ pH 7.0. The reconstituted protein was incubated at $30^{\circ} \mathrm{C}$ for $1 \mathrm{~h}$ and then complex protein was purified with gel filtration chromatography using Sephacryl-200 column (GE, Healthcare, USA) on an AKTA chromatography/protein purification workstation (GE Healthcare, USA). The reconstituted HqD complex(s) were subsequently analyzed for HqD activity using a spectrophotometric activity assay. The reconstituted HqD complex showing positive $\mathrm{HqD}$ activity (described later) was determined by comparing the elution volume in the gel filtration chromatography and its comparison with standard proteins with known molecular weights.

\section{HqD activity assay}

The purified complexes from gel filtration chromatography described above were analyzed for $\mathrm{HqD}$ activity; positive HqD activity was determined spectrophotometrically by monitoring transformation of $\mathrm{HQ}$ used as the reaction substrate into 4-HMS ( $\mathrm{Abs}_{\max }$ at $320 \mathrm{~nm}$; $\epsilon_{320}=11.0 \mathrm{mM}^{-1} \mathrm{~cm}^{-1}$ ) as reported previously (Spain and Gibson 1991). The reaction mixture typically contained $50 \mathrm{mM} \mathrm{NaHPO}_{4} @ \mathrm{pH} 7.5$ and $100 \mu \mathrm{M}$ $\mathrm{FeSO}_{4}$ and various concentrations of reconstituted $\mathrm{HqD}$. Reactions were started by adding $100 \mu \mathrm{M}$ hydroquinone prepared in dimethyl-formamide. One unit of $\mathrm{HqD}$ enzyme activity was defined as the amount of enzyme required for formation of $1 \mu \mathrm{mol}$ HMS per minute. Protein concentration was measured routinely using Bradford reagent (Sigma, Germany). The gel filtration analysis carried out to determine the molecular weight of the reconstituted HqD and its purification to homogeneity was performed on a Sephacryl-200 column using Akta-prime (GE-Amersham, USA).

\section{Analytical methods}

The HqD enzyme activity products were subjected to organic solvent extraction using equal volume of ethyl acetate thrice in neutral, acidic and alkaline $\mathrm{pH}$ and evaporated to dryness using a Rotavapour (Buchi, Switzerland). Extracted samples were re-suspended in $100 \mu \mathrm{l}$ of ethyl acetate and subjected to gas chromatographic (GC) analysis using AutoSystem XL Gas chromatograph (Perkin Elmer, USA) equipped with the flame ionization detector. The thermal profile used for GC analysis was as follows: constant temperature for injector, oven and detector at $250^{\circ} \mathrm{C}, 200^{\circ} \mathrm{C}$ and $280^{\circ} \mathrm{C}$ respectively. Subsequent qualitative analysis of the reaction product was carried out with Gas chromatography-mass spectrometry (GC-MS) using Shimadzu QP2010 (Shimadzu Scientific Instruments., USA). The identity of the reaction product was ascertained by mass fragmentation pattern and its comparison with the compound database NIST62LIB available on the GC-MS instrument.

\section{Induciblity of pnpE1 and pnpE2 with RT- PCR analysis}

Reverse transcription-Polymerase Chain Reaction (RT-PCR) analysis was carried out using a QIAGEN One-Step RT-PCR kit. Strain SJ98 was grown at $30^{\circ} \mathrm{C}$ overnight in $\mathrm{MM}$ with $20 \mathrm{mM}$ sodium succinate. The culture was induced with $0.3 \mathrm{mM}$ PNP once reached an $\mathrm{OD}_{600}$ of $\sim 0.5$ and was further incubated for $10-12 \mathrm{~h}$ at $30^{\circ} \mathrm{C}$. Reverse transcriptase PCR (RT-PCR) analyses were performed to amplify internal fragments of $p n p E 1$ and pnpE2 using primer pairs listed as pnpE1-RT_F, pnpE1RT_R and pnpE2-RT_F, pnpE2-RT_R respectively (Table 2) (232 bp and 182 bp respectively) and total cellular RNA isolated as described earlier. Amplicons obtained from RT-PCR were analyzed with $1.2 \%$ agarose gel electrophoresis. Total cellular RNA isolated from cells of strain SJ98 grown on MM with $20 \mathrm{mM}$ sodium succinate without PNP was used as a negative control.

\section{Nucleotide sequence accession number}

The nucleotide and amino acid sequence data reported in this paper have been deposited to the GenBank 
sequence database with accession numbers JN968480 and JN968481.

\section{Results}

Isolation of pnp gene cluster from strain SJ98

In order to isolate and clone genes responsible for PNP degradation by Burkholderia sp. strain SJ98, a cosmid genomic library was constructed. In an earlier study the use of a partial btd gene fragment ( 540 bp) as a probe to screen plasmid based genomic library has been described (Chauhan et al. 2010). The cosmid library was also screened using the same probe and a total of 8 positive cosmid clones (Additional file 1: Figure S1A and Additional file 1: Figure S1B). These positive clones were also verified by the amplification of $b t d$ specific DNA amplicon using colony PCR assay (Additional file 1: Figure S1C). Restriction digestion analysis was carried out to determine the length of the insert of the positive clones and found insert length in the range of 38 to $41 \mathrm{~Kb}$ (Additional file 1: Figure S1D). One of the positive clones designated as pSJC88 with insert size of $\sim 41 \mathrm{~Kb}$ was selected for sequencing and further characterization.

\section{Sequence annotation of pSJC88}

The result from sequencing of pSJC88 showed that insert of $\sim 41 \mathrm{~Kb}$ harbored a $6.8 \mathrm{~Kb}$ EcoRI fragment that hybridized with BtD (specific radio labeled DNA probe). Annotation of $6.8 \mathrm{~Kb}$ with additional $3 \mathrm{~Kb}$ on either side (upstream and downstream of this fragment) showed presence of 6 orfs, with each of these being transcribed in the same direction. Two of these 6 orfs were found to be those that were previously characterized as $p n p C$ and $p n p D$ which encode for MaR and BtD respectively. Upstream to $\mathrm{MaR}$, three other orfs viz., $p n p E 1, p n p E 2$ and $p n p F$ were identified. The former two showed highest sequence similarity with cognate (large and small) subunits of $\mathrm{HqD}$ whereas later one showed maximum sequence similarity with 4-hydroxymuconic semialdehyde dehydrogenase (4-HMSD). The BLAST search analyses indicated that pnpE1 shared $74 \%$ sequence identity with large subunit of HqD characterized from PNP degrading Pseudomonas sp. strain WBC-3 (accession number ABU50916.1), while pnpE2 had 59\% sequence identity with small subunit of $\mathrm{HqD}$ characterized from Pseudomonas sp. strain WBC-3 (accession number
ABU50917.1) and a hydroxyacetophenone degrading strain Pseudomonas fluorescens (accession number ACA50457.2). A summary of the homology searches carried out for $p n p E 1, p n p E 2$ and other orfs of $p n p$ gene cluster of strain SJ98 is presented in Table 3.

\section{Heterologous expression of pnpE1 and pnpE2}

ORFs pnpE1 and pnpE2 were cloned into pDSET17 and expressed as $\mathrm{N}$-terminal 6x His tagged recombinant proteins in E. coli BL21- AI (Invitrogen Inc. CA, USA). Cell free soluble lysates were initially examined for $\mathrm{HqD}$ activity without success. It was noticed that most of the overexpressed protein from clone expressing $p n p E 1$ was present in inclusion bodies. Therefore, insoluble fractions from this clone were subjected to urea denaturation followed by protein refolding. After refolding PnpE1 was purified with $\mathrm{Ni}-$ NTA affinity chromatography and analyzed with SDS PAGE analyses. SDS PAGE analysis of the purified proteins (PnpE1 and PnpE2) revealed expected molecular weight (40 kDa and $20 \mathrm{kDa}$ respectively) for these His-tagged proteins (Figure 2A). Purified soluble PnpE1 and PnpE2 were tested individually for $\mathrm{HqD}$ activity in the spectrophotometric assay, however, no activity was observed.

\section{Reconstitution of active HqD complex}

Since, activity assays carried out with individual subunits (PnpE1 or PnpE2) did not show any HqD activity; in subsequent activity assay, purified soluble subunits were mixed in different molecular ratios and multimeric complexes were purified over size exclusion chromatography. One of such multimeric complexes which eluted between $43-45 \mathrm{ml}$ on a Sephacryl-200 column (with predicted molecular weight of $\sim 120 \mathrm{kDa}$ ) was found to be positive for $\mathrm{HqD}$ activity in spectrophotometric assay. Figure 2B shows gel exclusion profiles of (i) PnpE2, (ii) PnpE1 and (iii) $\mathrm{PnpE} 1_{2}-\mathrm{PnpE} 2_{2}$ heterotetrameric complex. The positive $\mathrm{HqD}$ activity was observed with time dependent decrease in absorbance at $288 \mathrm{~nm}$ corresponding $\mathrm{Ab}_{\max }$ of the reaction substrate $\mathrm{HQ}$, and gradual increase in absorbance at $320 \mathrm{~nm}$ corresponding to 4-HMS (molar extinction coefficient $=11 \mathrm{mM}^{-1} \mathrm{Cm}^{-1}$ ) (Figure $\left.3 \mathrm{~A}\right) . \mathrm{GC}$ and GC-MS analyses also ascertained identity of the reaction product. The mass fragmentation pattern of reaction product obtained from the $\mathrm{HqD}$ activity assay showed presence of protonated pseudo molecular ion with $[\mathrm{m} / \mathrm{z}]$

Table 3 Amino acid sequence comparison of the ORFs identified in pnp gene cluster of Burkholderia sp. strain SJ98

\begin{tabular}{|c|c|c|c|c|c|c|}
\hline ORF & Similar protein & Predicted function & \% Identity & Score & E-value & Accession no. \\
\hline PnpC & PnpG & Hydroxyquinol dioxygenase, Pseudomonas sp. WBC-3 & 54 & 307 & $1 e-102$ & ABU50913.1 \\
\hline PnpD & HapF & Maleylacetate reductase, $P$. fluorescens & 60 & 424 & $1 e-146$ & ACA50460.1 \\
\hline PnpE1 & PnpD & Hydroquinone dioxygenase Large subunit, Pseudomonas sp. WBC-3 & 74 & 536 & 0.0 & ABU50916.1 \\
\hline PnpE2 & PnpC & Hydroquinone dioxygenase Small subunit, Pseudomonas sp. WBC-3 & 59 & 195 & $5 e-62$ & ABU50917.1 \\
\hline PnpF & PnpF & 4-hydroxymuconic semialdehyde dehydrogenase, Pseudomonas fluorescens & 73 & 761 & 0.0 & ACA50459.1 \\
\hline
\end{tabular}




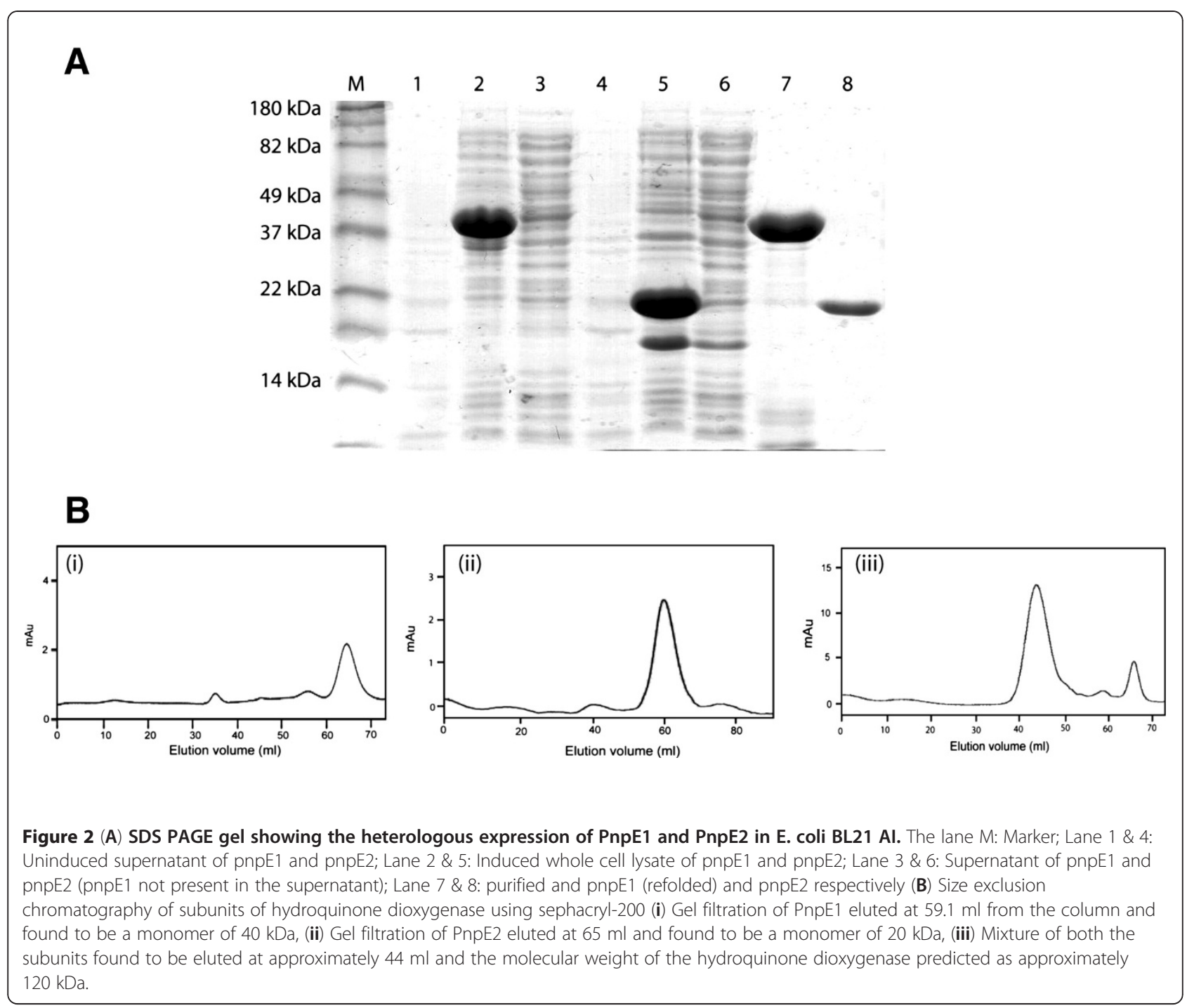

values of 142.04 (Additional file 1: Figure S2). The mass fragmentation pattern perfectly matched to the molecular weight of 4-HMS.

\section{Catalytic properties, substrate specificity and stability of $\mathrm{HqD}$ from strain $\mathrm{SJ} 98$}

Catalytic properties of $\mathrm{HqD}$ were routinely analyzed with spectrophotometric assay that monitors ring cleavage using HQ as reaction substrate and resulting in formation of 4-HMS (as described above). HqD activity was observed only in presence of $\mathrm{Fe}(\mathrm{II})$ and maximum activity was measured at $100 \mu \mathrm{M} \mathrm{FeSO} \mathrm{H}_{4}$. $\mathrm{HqD}$ from strain SJ98 shows a broad $\mathrm{pH}$ optima ranging from $\mathrm{pH} 7.0-$ 8.0 with maximum activity at $\mathrm{pH} 7.2$ (Figure $3 \mathrm{Bi}$ ). Optimal temperature for $\mathrm{HqD}$ activity was observed in a range of $25-30^{\circ} \mathrm{C}$ (Figure 3Bii). Kinetic analyses showed a Vmax of $9.85 \pm 0.4152 \mu$ mole $\min ^{-1} \mu \mathrm{g}^{-1}$ and $\mathrm{Km}$ of $65.32 \pm 10.49 \mu \mathrm{M}$ (at $\mathrm{pH} 7.0$ and $25^{\circ} \mathrm{C}$ ) as determined from the Michaelis-Menten plot for kinetic analyses (Figure 3Biii). Reconstituted HqD catalyzed ring cleavage in a substrate specific manner with only HQ as the reaction substrate. Neither substitute hydroquinones (e.g. Chlorohydroquinone) nor other biphenolic compounds (e.g. catechol, resorcinol, chlorocatechol and nitrocatechol) were used as the reaction substrate by this enzyme (data not shown). In presence of ferrous ion chelator e.g. 2,2'-dipyridyl, the activity of $\mathrm{HqD}$ from strain SJ98 was completely abolished. The inactivation of enzyme activity appeared irreversible since it could not be restored by extended incubation at lower temperature with or without $\mathrm{Fe}(\mathrm{II})$. Rapid and complete inactivation of reconstituted $\mathrm{HqD}$ also occurred upon incubation with $100 \mu \mathrm{M}$ hydrogen peroxide. Incubation at $40^{\circ} \mathrm{C}$ for $30 \mathrm{~min}$ and $45^{\circ} \mathrm{C}$ for $15 \mathrm{~min}$ also resulted in complete inactivation of the $\mathrm{HqD}$ activity. 


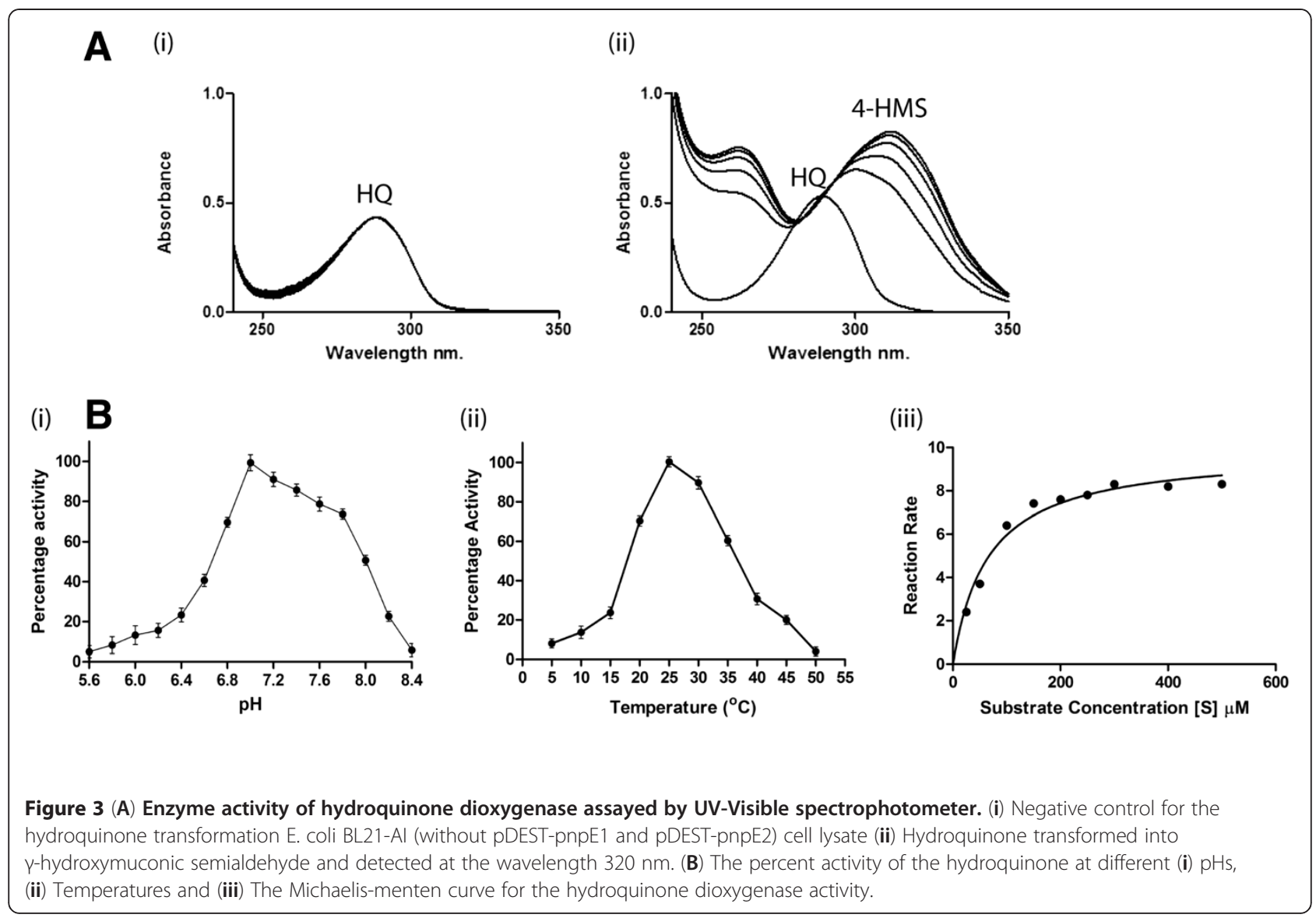

\section{Induction of pnpE1 and pnpE2 in strain SJ98 during growth on PNP}

It is important to understand whether $p n p E 1$ and $p n p E 2$ are selectively induced during growth of strain SJ98 on PNP. DNA-free total cellular RNA isolated from cells of strain SJ98, grown with and without PNP. These isolates were analyzed using RT-PCR, targeting transcripts corresponding to the above genes. In this analysis, the primer pairs were designed to amplify internal fragments corresponding to $p n p E 1$ and $p n p E 2$ transcript. RT-PCR products of expected size could be successfully amplified using RNA purified from SJ98 culture grown on PNP but not from cultures grown without PNP (Additional file 1: Figure S3). The RT- PCR products were also not observed when chemical analogues of HQ e.g. 2chlorohydroquinone, 2-methylhydroquinone or intermediates involved in the 'BT' cleavage pathway e.g. 4-NC and BT were used as inducer during cell growth of strain SJ98 (data not shown). These results provide strong indication for involvement of HqD is involved in PNP degradation process.

\section{Discussion}

Genetic characterization of gene(s) and regulatory elements involved in degradation of xenobiotic compounds is extremely important for successful development of bioremediation technologies as well as for basic understanding of biochemical and molecular mechanisms of degradation process. Hitherto, a number of studies have reported cloning and characterization of PNP degradation gene clusters. During present study, we cloned, sequenced and characterized a PNP degrading DNA fragment from Burkholderia sp. strain SJ98. The sequence analyses and annotation of this fragment indicated that the organization of pnp gene cluster in strain SJ98 is very similar to that of the nph gene cluster of Arthrobacter sp. strain JS443 (Perry and Zylstra 2007). However, the only striking difference is the presence of two orfs within pnp cluster of strain SJ98 that share strong sequence similarity with genes encode for cognate subunits of HqD. During earlier studies, we detected HQ as a transient metabolite in PNP degradation by strain SJ98; however, in absence of genetic information, HQ could not be ascertained as a true degradation intermediate. Instead, it was suggested that strain SJ98 may non- specifically transform PNP into $\mathrm{HQ}$ as a dead end product which does not contribute to the active catabolism of PNP. Similar observation has also been reported with PNP degradation by strain JS443 
(Jain et al. 1994; Perry and Zylstra 2007). Results obtained during the present study and previous study with strain SJ98 clearly establish presence of a functional genes encoding for enzymes involved in both the branches of PNP degradation (viz., HqD and BtD) within the PNP degradation gene cluster of strain SJ98. Characterization of $\mathrm{HqD}$ carried out during present study established that this enzyme catalyzes Fe(II) dependent transformation of $\mathrm{HQ}$ to 4-HMS. This observation indicates that HqD from strain SJ98 belongs to 'Ferrous ion dependent type II hydroquinone dioxygenase. Also, the active reconstituted HqD complex as predicted on the basis of the molecular weights of complex, PnpE1 and PnpE2 was found to be a heterotetrameric complex consisting of 2 molecules of both subunits. These observations are in agreement with earlier reports of $\mathrm{HqD}$ from other microorganisms. Ferrous ion dependent, heterotetrameric HqD complex has been previously reported to catalyze ring cleavage of HQ formed during degradation of hydroxyacetophenone (Moonen et al. 2008) and PNP (Shen et al. 2010; Zhang et al. 2009). The apparent molecular weight of reconstituted HqD from strain SJ98 $(\sim 120 \mathrm{kDa})$ is also within the range of molecular weights $(112 \mathrm{kDa}-$ $120 \mathrm{kDa}$ ) of other reported HqDs (Shen et al. 2010; Spain and Gibson 1991; Moonen et al. 2008; Kolvenbach et al. 2011). Noticeably, HqD from strain SJ98 also showed stringent substrate specificity in the activity assay and is selectively induced during growth of strain SJ98 on PNP; these observations clearly indicate it to be involved in transformation of HQ produced during PNP catabolism by strain SJ98. Conspicuously, stringent substrate specificity has not been observed with $\mathrm{HqDs}$ characterized from Sphingomonas sp. strain TTNP-3, Pseudomonas fluorescens strain ABC, Cupriavidus necator JMP134 and Pseudomonas sp. strain NyZ402 (Moonen et al. 2008; Kolvenbach et al. 2011; Yin and Zhou 2010; Wei et al. 2010b). The reason and molecular mechanism for such different behavior is unclear and is still under investigation.

Based on the results presented above, we propose orf $p n p \mathrm{E} 1$ and $p n p \mathrm{E} 2$ as genes encoding for functional cognate subunits of $\mathrm{HqD}$ that catalyze transformation reaction involved in HQ branch. Previously, orfs pnpC and pnpD were characterized as genes encoding for $\mathrm{BtD}$ and $\mathrm{MaR}$ that are involved in $\mathrm{BT}$ branch of PNP degradation pathway. Based on these results we show a graphical representation of the branching organization of $p n p$ gene cluster from strain SJ98 (Figures 1A and B). Results obtained from RT-PCR analyses targeting mRNA transcripts corresponding to pnpE1 and pnpE2 in strain SJ98 cells grown with PNP demonstrated PNP specific induction of these genes. Previously, Chauhan et al. (2010) detected $\mathrm{BtD}$ and MaR specific transcripts in PNP induced cells of strain SJ98 indicating their inducible nature. Together these results present conclusive evidence for branching of PNP degradation pathway in any PNP degrading isolate. Similar observation was reported by Zhang et al. in Pseaudomonas sp. stain 1-7 where activity of four PNP degradation genes ( $p d c D E F G$ ) expressed in heterologous system in vitro, supports the existence of HQ and BT mediated degradation pathways (Zhang et al. 2012).

Few earlier studies on genetic characterization of PNP degrading Pseudomonads viz., Pseudomonas sp. strain WBC and Pseudomonas putida strain DLL-E4 also reported the presence of both $\mathrm{BtD}$ and $\mathrm{HqD}$ within the same genetic locus (Shen et al. 2010; Wei et al. 2010a; Zhang et al. 2009). However, the PNP degradation in these strains proceeds only via HQ ring cleavage pathway and utilizes $\mathrm{HqD}$ whereas the BT cleavage enzyme $\mathrm{BtD}$ is only selectively utilized in 4-NC degradation (Kitagawa et al. 2004). Similar involvement for both HQ and BT branches of degradation has been reported in the degradation of 4-chlorophenol (4-CP) by A. chlorophenolicus A6 (Nordin et al. 2005). Nordin et al. (2005) reported involvement of two pathways but the terminal aromatic ring cleavage intermediate is the hydroxyquinol (Nordin et al. 2005). One branch of degradation of 4-CP by $A$. Chlorophenolicus A6 is proceeds via the hydroquinone and then finally into the hydroxyquinol whereas the other branch of this pathway ocuurs via the conversion of 4-CP to chlorohydroxyquinol and finally into the hydroquinone which subsequently transformed into hydroxyquinol (Nordin et al. 2005; Unell et al. 2007; Unell et al. 2009).

The annotated pnp gene cluster of Strain SJ98 also indicates presence of other genes required for the respective pathways. Further, studies for heterologous expression, purification, and biochemical characterization of other genes are currently underway to rigorously demonstrate involvement of both the pathways. Results obtained with biochemical characterization of purified $\mathrm{HqD}$ during the present study clearly demonstrate that HqD from strain SJ98 is a Fe(II) requiring, type II, heterotetrameric $\mathrm{HqD}$ and it represents the first enzyme of this type characterized from a member of the genus Burkholderia. It is actively involved in catabolic degradation of PNP by strain SJ98. Furthermore it gets selectively induced during growth of strain SJ98 on PNP. Data gathered in this study and also supported by our previous findings present conclusive evidence for cooccurrence of both 'HQ and BT' ring cleavage branches of PNP degradation pathway in strain SJ98. We propose Burkholderia sp. strain SJ98 as an important model system for further studies on evolution of PNP degradation pathways. 


\section{Additional file}

\section{Additional file 1: Screening of cosmid library clones (A) colony} hybridization blots were hybridized with the 540 bp partial benzenetriol dioxygenase gene. Blot $A$ represents preliminary round of screening and (B) blot B represents secondary phase of screening where only the suspected colonies were used for blotting. (+) represents the position of positive control (genomic DNA of strain SJ98). (-) represents the position of negative control (genomic DNA of E. coli DH5a). Arrows indicate hybridization signals with the probe; (C) Colony PCR amplification of partial (540 bp) and complete benzenetriol dioxygenase gene (818 bp) from one of the clones (Lanes 1, 2). Amplification of aldehyde dehydrogenase gene from the same clone (Lane 3). Lanes 4, 5, 6 show amplification of the same gene(s) under the same condition as with the positive control (pSJC262). Lane M represents 1 kb ladder; (D) The restriction pattern of cosmid clones no. 957 (Lane 2), 946 (Lane 3), 1956 (Lane 4), 1881 (Lane 5), 88 (Lane 6), 1 kb ladder (Lane M1), $\lambda$ HindIII digest ladder (Lane M2).

\section{Competing interests}

The authors declare that they have no competing interests.

\section{Acknowledgements}

This study was carried out in part with financial support from Council for Scientific and Industrial Research (CSIR)-India, and Indo-Australian collaboration in Biotechnology (IACB). SV, JP, AG and FK acknowledge the research fellowship granted by CSIR India. We are thankful to Ms. Archana Chauhan for suggestion during course of the study and to Mr. Dhan Prakash for his technical help in carrying out some of the experiments. This is the IMTECH communication number 085/2011.

\section{Author details}

${ }^{1}$ Institute of Microbial Technology (CSIR), Sector 39 A, Chandigarh 160036, India. ${ }^{2}$ Georgia Health Sciences University, Augusta, GA 30912, USA. ${ }^{3}$ CSIROEco Systems Sciences, Clunies Ross Street, Acton ACT-2601, Australia. ${ }^{4}$ Kansas State University, Manhattan, KS 66506, USA.

Received: 18 April 2012 Accepted: 24 April 2012

Published: 8 June 2012

\section{References}

Bhushan B, Chauhan A, Samanta SK, Jain RK (2000) Kinetics of biodegradation of $\mathrm{p}$-nitrophenol by different bacteria. Biochem Biophys Res Commun 274:626-630

Chauhan A, Chakraborti AK, Jain RK (2000) Plasmid-encoded degradation of p-nitrophenol and 4-nitrocatechol by Arthrobacter protophormiae. Biochem Biophys Res Commun 270:733-740

Chauhan A, Pandey G, Sharma NK, Paul D, Pandey J, Jain RK (2010) p-Nitrophenol degradation via 4-nitrocatechol in Burkholderia sp. SJ98 and cloning of some of the lower pathway genes. Environ Sci Technol 44:3435-3441

Eppink MH, Cammaart E, Van Wassenaar D, Middelhoven WJ, van Berkel WJ (2000) Purification and properties of hydroquinone hydroxylase, a FADdependent monooxygenase involved in the catabolism of 4hydroxybenzoate in Candida parapsilosis CBS604. Eur J Biochem 267:6832-6840

Jain RK, Dreisbach JH, Spain JC (1994) Biodegradation of p-nitrophenol via 1,2,4benzenetriol by an Arthrobacter sp. Appl Environ Microbiol 60:3030-3032

Kadiyala V, Spain JC (1998) A two-component monooxygenase catalyzes both the hydroxylation of $p$-nitrophenol and the oxidative release of nitrite from 4-nitrocatechol in Bacillus sphaericus JS905. Appl Environ Microbiol 64:2479-2484

Kitagawa W, Kimura N, Kamagata Y (2004) A novel p-nitrophenol degradation gene cluster from a gram-positive bacterium, Rhodococcus opacus SAO101. J Bacteriol 186:4894-4902

Kolvenbach BA, Lenz M, Benndorf D, Rapp E, Fousek J, Vlcek C, Schäffer A, Gabriel FL, Kohler HP, Corvini PF (2011) Purification and characterization of hydroquinone dioxygenase from Sphingomonas sp. strain TTNP3. AMB Express 1:8
Liu H, Zhang JJ, Wang SJ, Zhang XE, Zhou NY (2005) Plasmid-borne catabolism of methyl parathion and p-nitrophenol in Pseudomonas sp. strain WBC-3. Biochem Biophys Res Commun 334:1107-1114

Moonen MJ, Synowsky SA, van den Berg WA, Westphal AH, Heck AJ, van den Heuvel RH, Fraaije MW, van Berkel WJ (2008) Hydroquinone dioxygenase from Pseudomonas fluorescens ACB: a novel member of the family of nonheme-iron(II)-dependent dioxygenases. J Bacteriol 190:5199-5209

Nordin K, Unell M, Jansson JK (2005) Novel 4-chlorophenol degradation gene cluster and degradation route via hydroxyquinol in Arthrobacter chlorophenolicus A6. Appl Environ Microbiol 71:6538-6544

Paul D, Rastogi N, Krauss U, Schlomann M, Pandey G, Pandey J, Ghosh A, Jain RK (2008) Diversity of 'benzenetriol dioxygenase' involved in $p$-nitrophenol degradation in soil bacteria. Indian J Microbiol 48:279-286

Perry LL, Zylstra GJ (2007) Cloning of a gene cluster involved in the catabolism of p-nitrophenol by Arthrobacter sp. strain JS443 and characterization of the p-nitrophenol monooxygenase. J Bacteriol 189:7563-7572

Samanta SK, Bhushan B, Chauhan A, Jain RK (2000) Chemotaxis of a Ralstonia sp. SJ98 toward Different Nitroaromatic Compounds and Their Degradation. Biochem Biophys Res Commun 269:117-123

Shen W, Liu W, Zhang J, Tao J, Deng H, Cao H, Cui Z (2010) Cloning and characterization of a gene cluster involved in the catabolism of p-nitrophenol from Pseudomonas putida DLL-E4. Bioresour Technol 101:7516-7522

Spain JC (1995) Biodegradation of nitroaromatic compounds. Annu Rev Microbiol 49:523-555

Spain JC, Gibson DT (1991) Pathway for Biodegradation of $p$-Nitrophenol in a Moraxella sp. Appl Environ Microbiol 57:812-819

Takeo M, Murakami M, Niihara S, Yamamoto K, Nishimura M, Kato D, Negoro S (2008) Mechanism of 4-nitrophenol oxidation in Rhodococcus sp. Strain PN1: characterization of the two-component 4-nitrophenol hydroxylase and regulation of its expression. J Bacteriol 190:7367-7374

Unell M, Abraham PE, Shah M, Zhang B, Ruckert C et al (2009) Impact of phenolic substrate and growth temperature on the Arthrobacter chlorophenolicus proteome. J Proteome Res 8:1953-1964

Unell M, Nordin K, Jernberg C, Stenstrom J, Jansson JK (2007) Degradation of mixtures of phenolic compounds by Arthrobacter chlorophenolicus A6. Biodegradation 19:495-505

Wei M, Zhang JJ, Liu H, Zhou NY (2010a) para-Nitrophenol 4-monooxygenase and hydroxyquinol 1,2-dioxygenase catalyze sequential transformation of 4-nitrocatechol in Pseudomonas sp. strain WBC-3. Biodegradation 21:915-921

Wei Q, Liu H, Zhang JJ, Wang SH, Xiao Y, Zhou NY (2010b) Characterization of a para-nitrophenol catabolic cluster in Pseudomonas sp. strain NyZ402 and construction of an engineered strain capable of simultaneously mineralizing both para- and ortho-nitrophenols. Biodegradation 21:575-584

Yin Y, Zhou NY (2010) Characterization of MnpC, a hydroquinone dioxygenase likely involved in the meta-nitrophenol degradation by Cupriavidus necator JMP134. Curr Microbiol 61:471-476

Zhang JJ, Liu H, Xiao Y, Zhang XE, Zhou NY (2009) Identification and characterization of catabolic para-nitrophenol 4-monooxygenase and para-benzoquinone reductase from Pseudomonas sp. strain WBC-3. J Bacteriol 191:2703-2710

Zhang S, Sun W, Xu L, Zheng X, Chu X, Tian J, Wu N, Fan Y (2012) Identification of the para-nitrophenol catabolic pathway, and characterization of three enzymes involved in the hydroquinone pathway, in Pseudomonas sp. 1-7. BMC Microbiol 12:27

Zheng Y, Liu D, Liu S, Xu S, Yuan Y, XIONG L (2009) Kinetics and mechanisms of p-nitrophenol biodegradation by Pseudomonas aeruginosa HS-D38. J Environ Sci 21:1194-1199

doi:10.1186/2191-0855-2-30

Cite this article as: Vikram et al:: Branching of the $p$-nitrophenol (PNP) degradation pathway in burkholderia sp. Strain SJ98: Evidences from genetic characterization of PNP gene cluster. AMB Express 2012 2:30. 